\title{
Manuscript Preparation Guidelines
}

Please review our Statement of Faith at http://www.xulonpress.com/about-xulon-press/statement-of-faith.php

If you feel God has given you a message to put in print, we are excited to help you get it published. We want to be your partner in self-publishing and spreading the Christian message through your book.

It is important that you use the following guidelines when submitting your manuscript to us for editing and/or formatting. Any variation to these guidelines may cause a delay.

\section{Professional Typesetting}

The interior text of your book will be professionally typeset according the Chicago Manual of Style. Using a printing standard software, we layout text and illustrations, to a specified design to produce a finished document ready for printing. If you desire any layout different than is specified in these guidlines please include "notes to the typesetter". However, Xulon Press reserves the right to determine the manner and style in which the book is formatted and typeset based on the styles that are consistent with popular trade books. Xulon Press DOES NOT guarantee it will perform any special or unusual formatting requests.

\section{File Types Accepted}

Please submit your final manuscript as ONE singe file in Microsoft Word format (.doc or .docx)

\section{Page Counts}

Keep in mind that your chosen trim size, font size and line spacing will affect the final page count of your book. In most cases your completed text will not match your manuscript's page count. Please note:

- Your finished book cannot exceed 1200 pages

- It must have at least 18 finished pages.

- If you require text on the spine your finished book must have at least 80 pages.

\section{Manuscript Content}

Submit your entire manuscript as ONE complete document, including front matter, chapters and end matter. Make sure content is in the order that you wish it to appear in the book. PLEASE NOTE: If content is added after the typeset is complete there may be an additional re-formatting charge.

FRONT MATTER Consists of: (include if applicable)

- Title Page - Including the following information: Title, Subtitle (if used) \& Pen Name

- Copyright Page (We will add pertinent and necessary verbiage to your copyright page, however, for any Scripture which is quoted please provide the Bible version used, i.e. NIV, KJV, NASB, etc. or if no Scripture is quoted indicate by stating "No Bible Information Needed.")

- Table of Contents (DO NOT include leader dots or page numbers)

- Introduction

- Dedication

- Acknowledgements

- Endorsements 
END MATTER Consists of (include if applicable):

- Work Cited or Bibliography

- About the Author

- Further Reading

- End Notes (We cannot guarantee that auto-format functions in MS Word will convert properly when we typeset your book. Please enter endnotes manually.)

- Index (Xulon Press is not responsible for adding page numbers to your index. Please indicate that you will need an index, but wait to submit the page numbers until after the final typeset is complete.)

Important Text Set-Up Information

- Fonts: When setting up your manuscript please use a standard font, such as Times New Roman, Times, or Arial. Bold, italics, underlines, etc. will be maintained when typeset by Xulon. Please note that some stylized fonts do not support bold, bold italic, etc. For your professional typeset you will be able to select from several standard and easy-to-read fonts for the body of your text.

- Headers \& Footers: Added during typesetting, DO NOT add them to your manuscript.

- Drop Caps : (Large letters at the beginning of chapters) Added during typesetting.

- Em Dashes: If you need to include em dashes type two hyphens and we will convert them to emdashes, or you can use the em function in MS word.

- Paragraphs : All paragraphs will be indented during typesetting. Do not use spaces to indent in your manuscript. Also, we will NOT keep spaces between paragraphs unless requested to keep them in Notes to the Typesetter.

- Hard Returns / Enter Key: Please do not use the Enter/Return key at the end of each line (as you would on a typewriter). This is called a "Hard Return". Let the text automatically flow from one line to the next. Using a hard return will result in a new paragraph when the text is flowed into our typesetting software.

- Section Breaks: Please indicate sections and/or chapter breaks clearly with several paragraph returns or with page breaks. DO NOT use the section break option in MS Word.

- Glyphs or Dingbats: (The decorative ornament at the beginning of each chapter) will be chosen by your typesetter to compliment your book.

- Pull Quotes: (Please do not add designed pull quotes to the manuscript). Submit a separate document with desired pull quote text - we will search the manuscript for that quote and add it to the same page OR when setting up your manuscript indicate in the text with $<$ Begin pullquote $>$ [text] $<$ End pullquote $>$. Be sure to alert the typesetter that you have pull quotes in Notes to the Typesetter.

- Hebrew/Greek characters: Please use specific Hebrew and/or Greek fonts and do not use special characters found in MS Word. You must submit the actual font files (Hebrew/ Green ONLY) via the Author Center with your submission. NOTE: if you have an ebook included in your package please be advised that Xulon cannot guarantee that these special fonts/characters will display correctly on ebook devices.

- Hyphens: DO NOT manually insert hyphens to indicate where a word should break.

- Tables / Charts / Textboxes: Considered to be graphics, however, though other images cannot be embedded in the manuscript, these should be included in the submitted manuscript. Please use text between 11 pt - 16pt. within tables, charts and textboxes. 
Graphic Submission Guidelines

- 50-graphic limit: Graphics include: pictures, icons, elements, graphs, charts, tables, text boxes, pull-out quotes, borders, etc. Anything that is not straight text is considered a graphic.

- Graphic Submission:

FOR BEST FINAL QUALITY SUBMIT GRAPHIC FILES SEPARATELY, NOT EMBEDDED IN THE MANUSCRIPT, except for charts and tables.

$>$ Photos/lmages - $300 \mathrm{dpi}$ or higher, .Tif (preferred for best quality), .jpg or .pdf. Submit them at the size they will be in print.

- CMYK for Color books

- Grayscale for black and white books, (Color images can be submitted for black and white books to be converted to grayscale).

$>$ Icons/Elements - Vector Format or .eps files for best quality.

$>$ Charts/Tables - CAN BE EMBEDDED in the Word Doc manuscript or submit as .pdf

- Naming Image Files: Name each Image simply as in “Image01.tif" or "01.jpg", etc. in the order they will appear in the book. (NO spaces, long names, such as "Billy and Suzie playing ball in the yard.tif", or page numbers, such as "Page 10.jpg".

- Image Placement and Captions: Please indicate in your manuscript where you would like your images to be placed. Use the appropriate image name and caption. (Example: "[PLACE Image01.tif HERE - CAPTION: Aunt Mary and Uncle Bob 1999]".

\section{What to Expect (When you receive your typeset galley)}

- We will typeset your book using standard publishing formatting guidelines.

- All front and back matter will start on the right side (odd pages) of your book. Remaining chapters will fall consecutively on either the right or left side (unless your book has only a few pages or chapters, or if requested to start all chapters on the right).

- Blank pages will be added as deemed necessary by standards, including a required blank page at the end of the book for printer information and ISBN number.

- All double-spaces (2 space-bar hits) after periods will be converted to single spaces as is the current standard.

- All line-spacing between paragraphs will be taken out - with paragraphs starting with firstline indents. (Unless requested differently in Notes to the Typesetter.)

The following link will take you to a sample of the industry standard layout. If your manuscript requires specific formatting that differs from the sample, pleas note this in a Notes to the Typesetter or in the comments section of your Author Center during the submission process.

\section{$\underline{\text { Industry Standard Sample }}$}

For further questions regarding these guidelines, please contact your Customer Service Representative toll-free at 1-866-381-2665. 


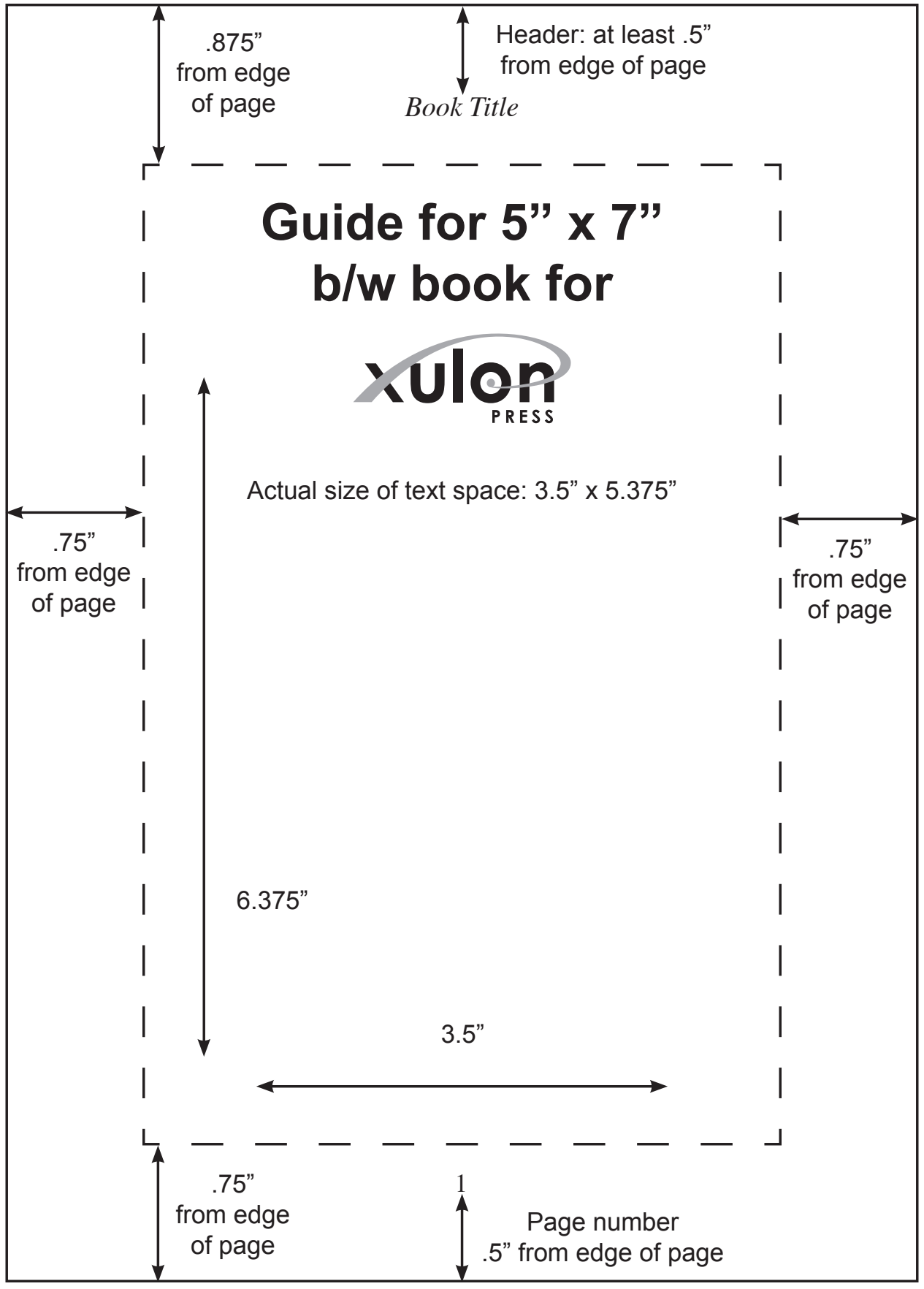




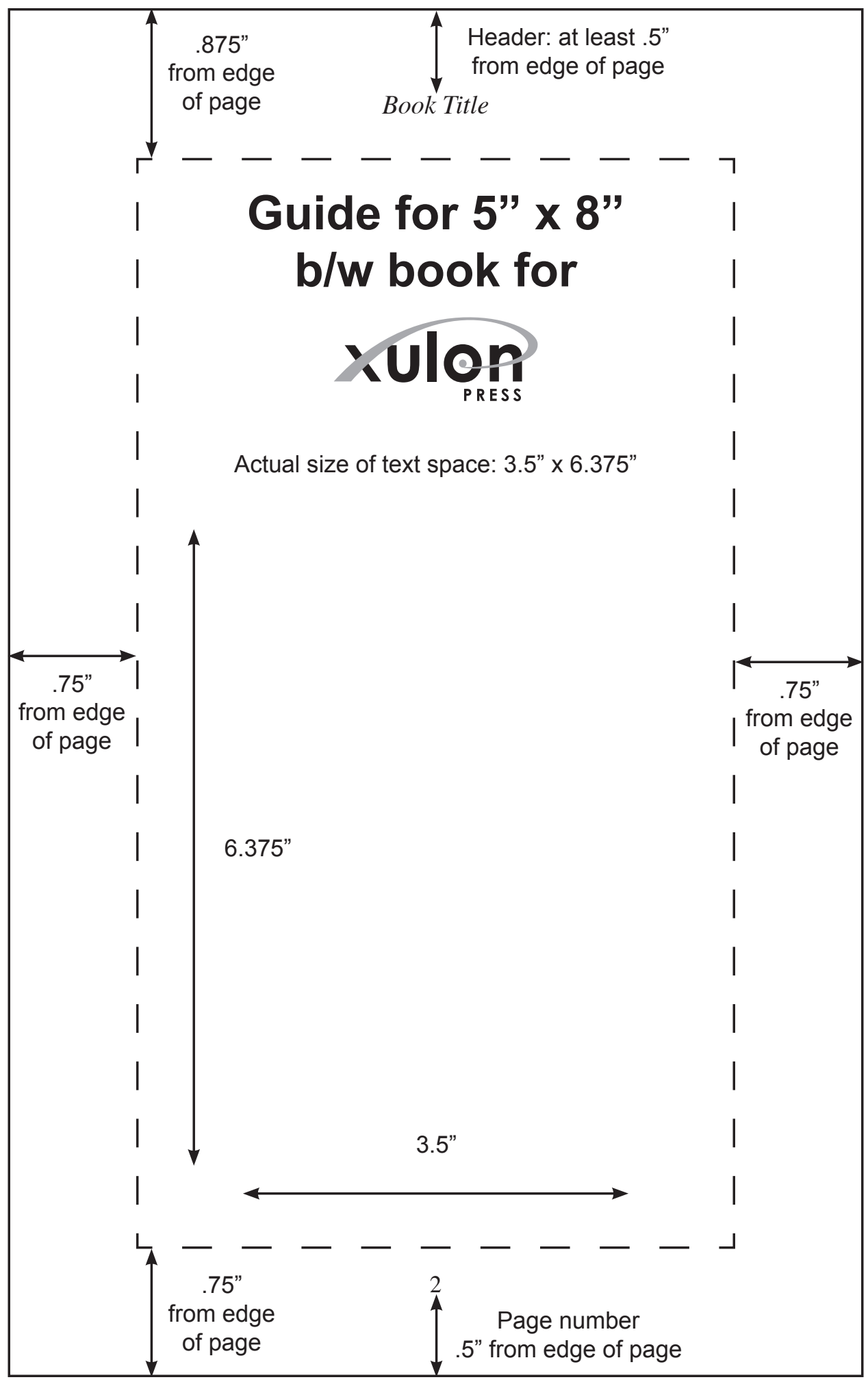




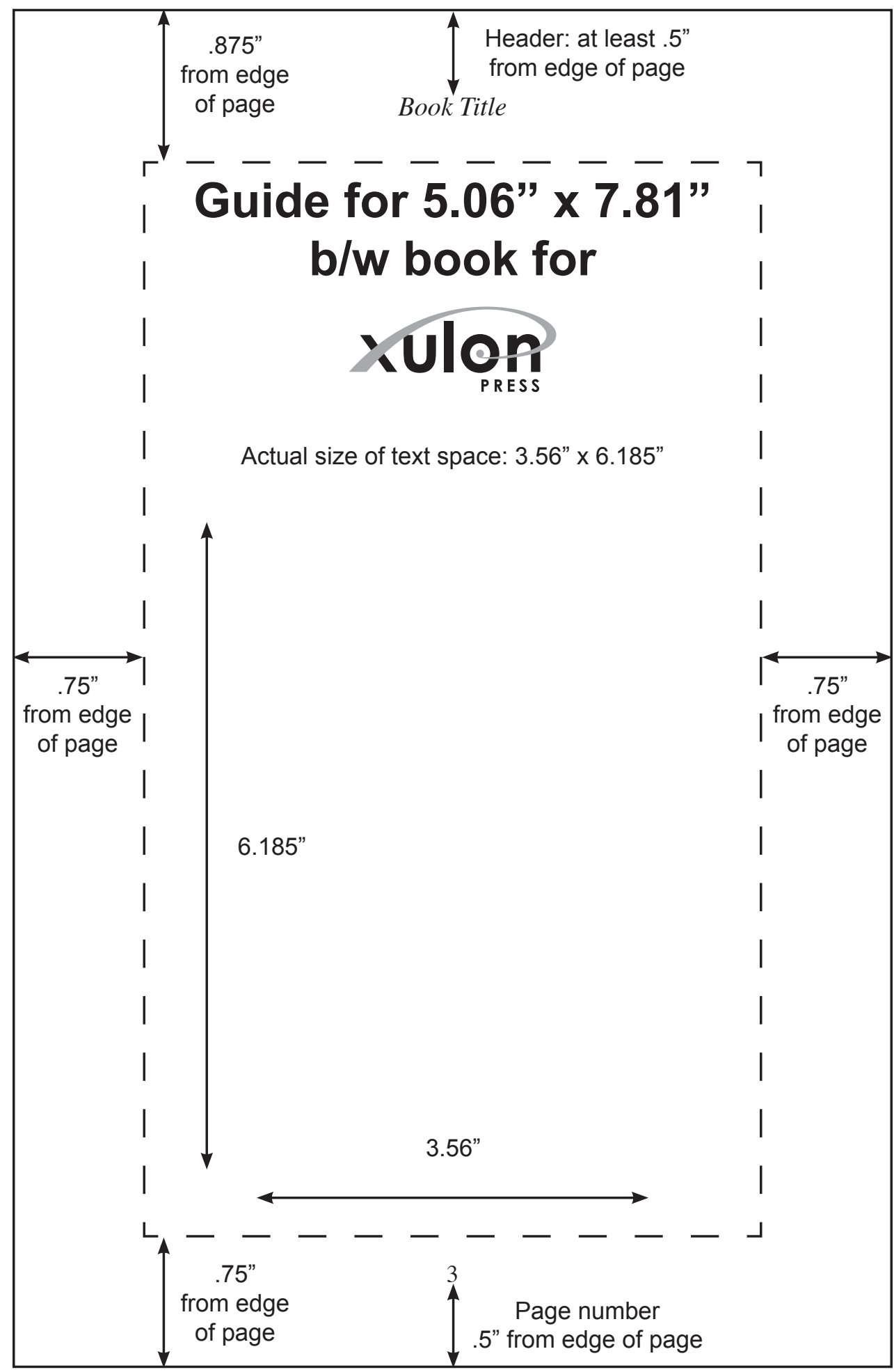




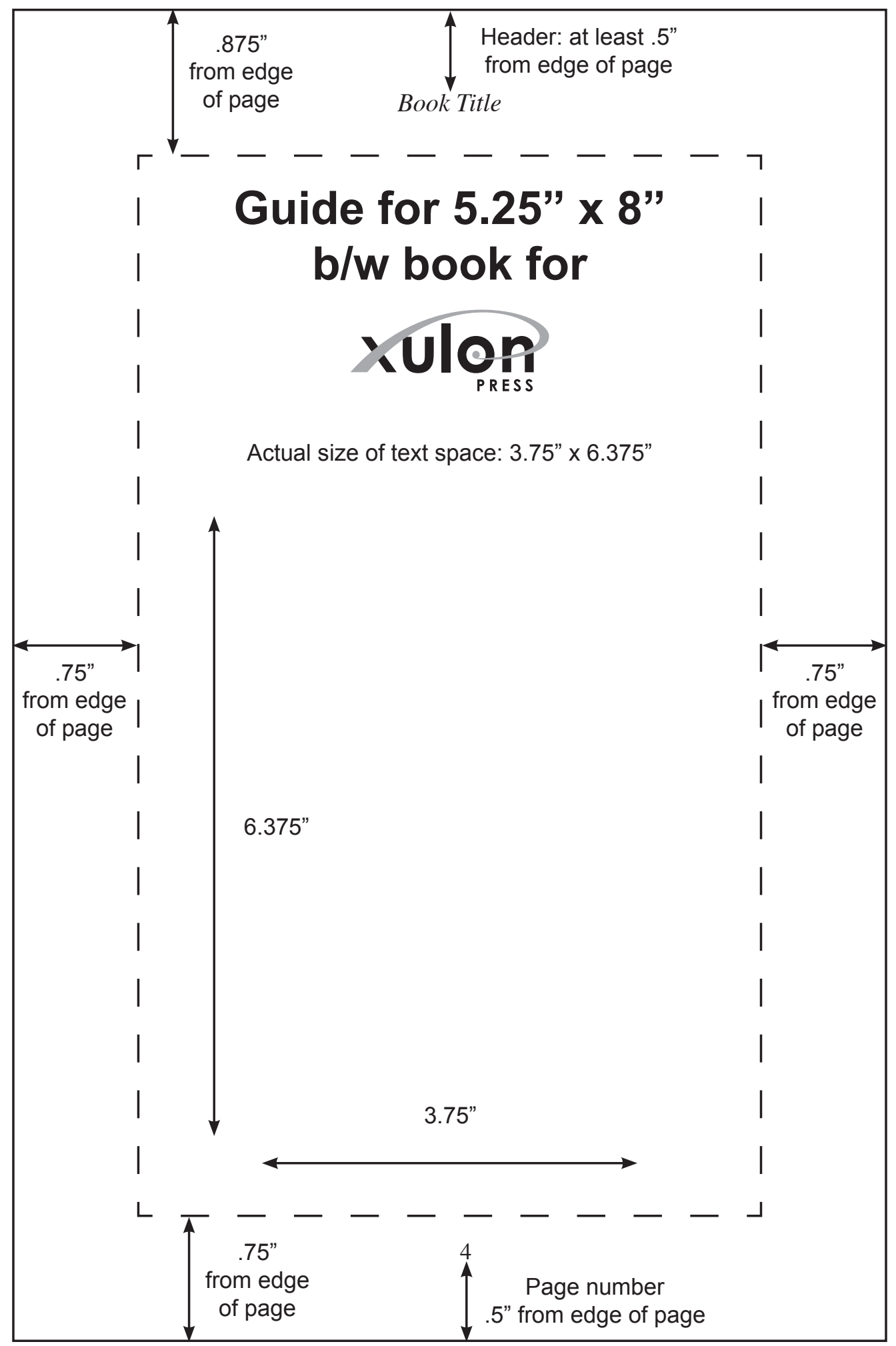




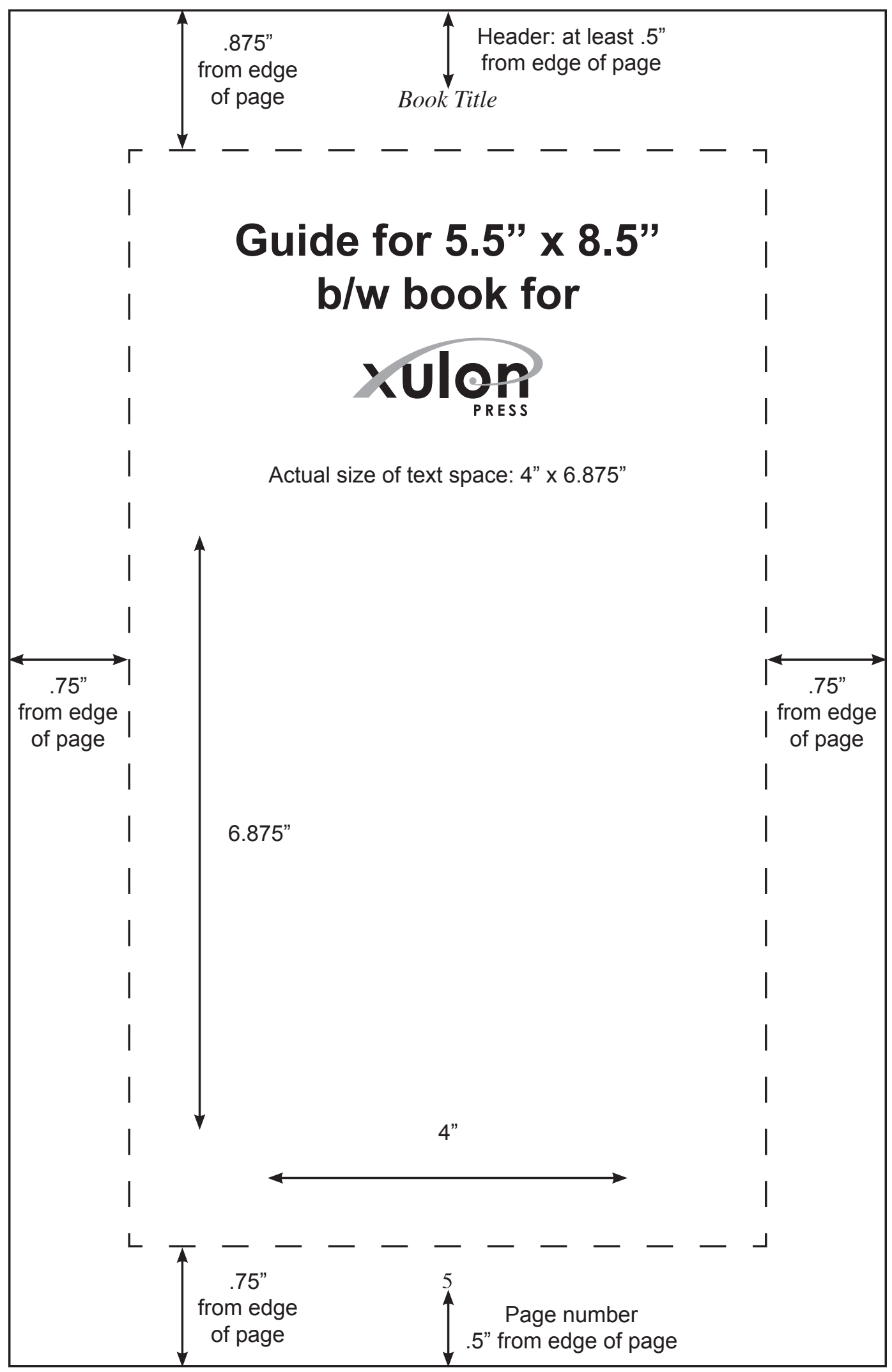




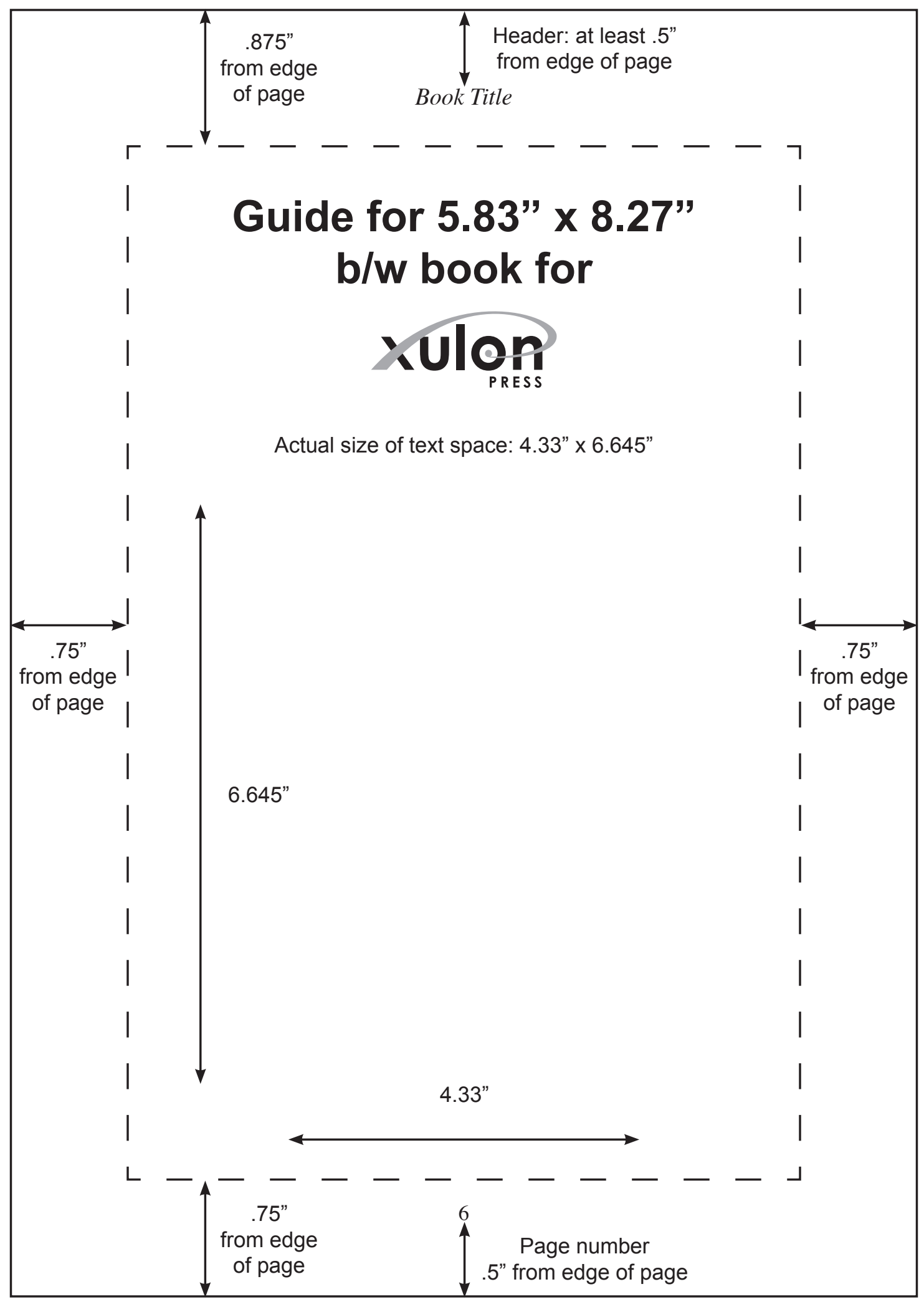




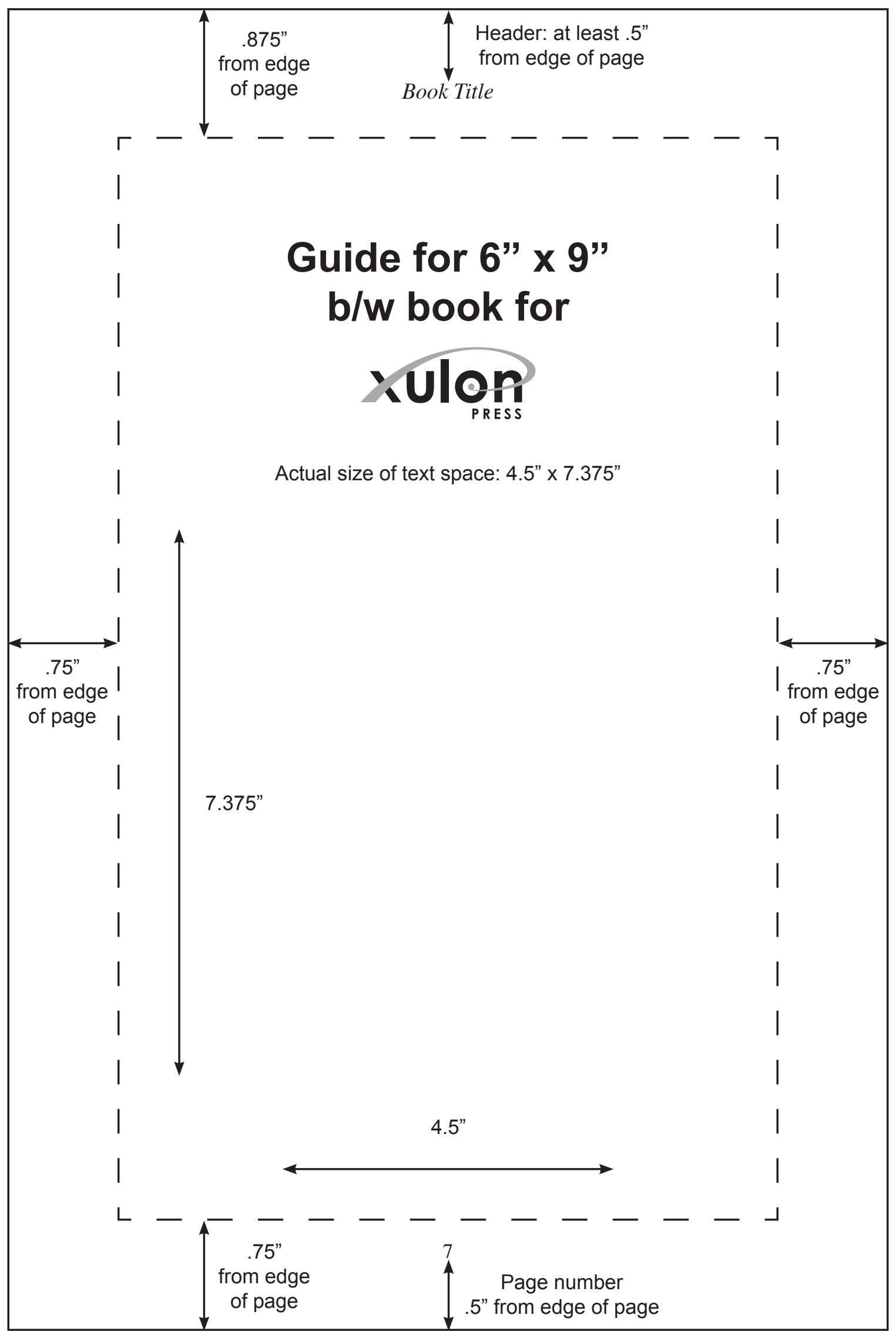




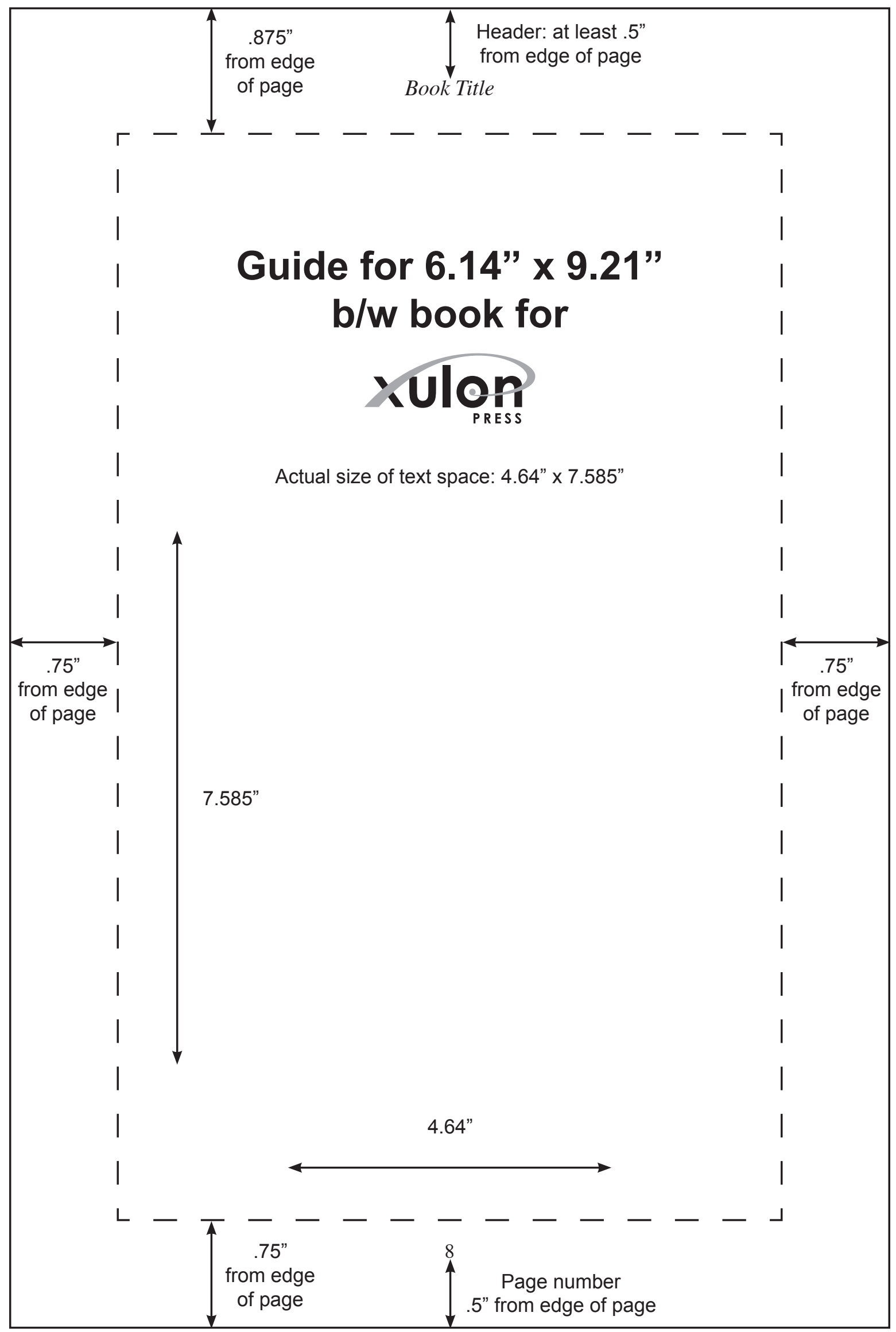




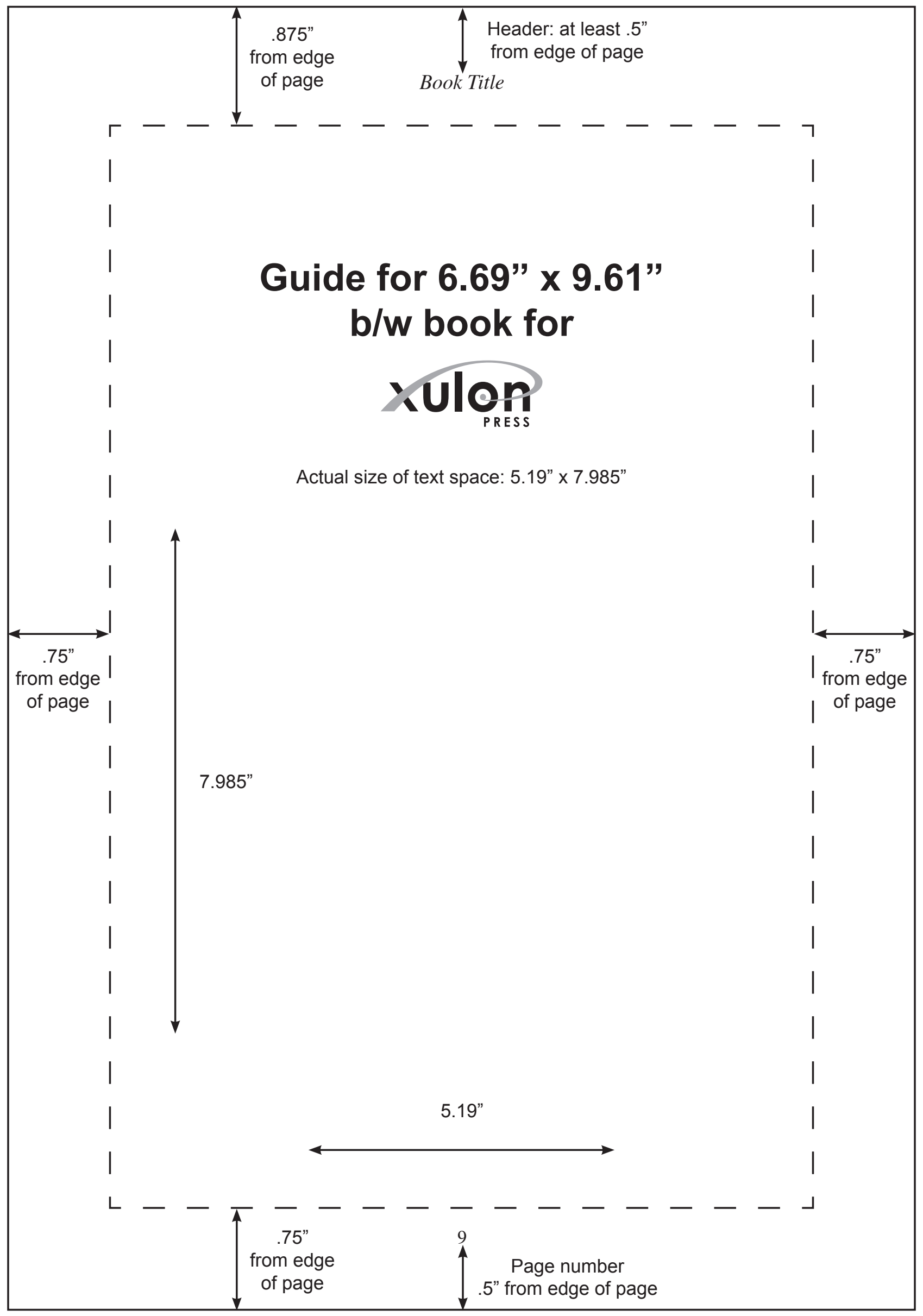




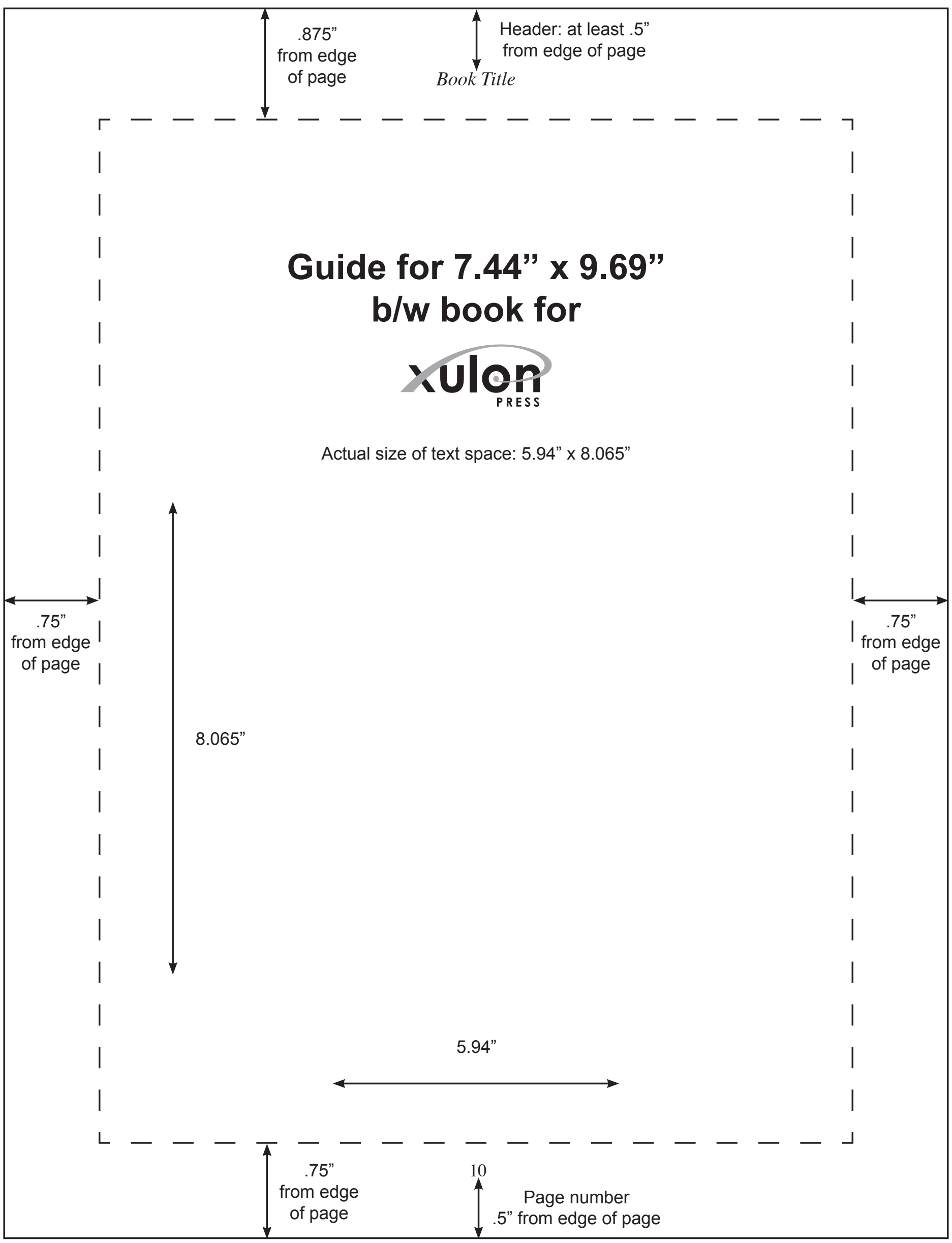




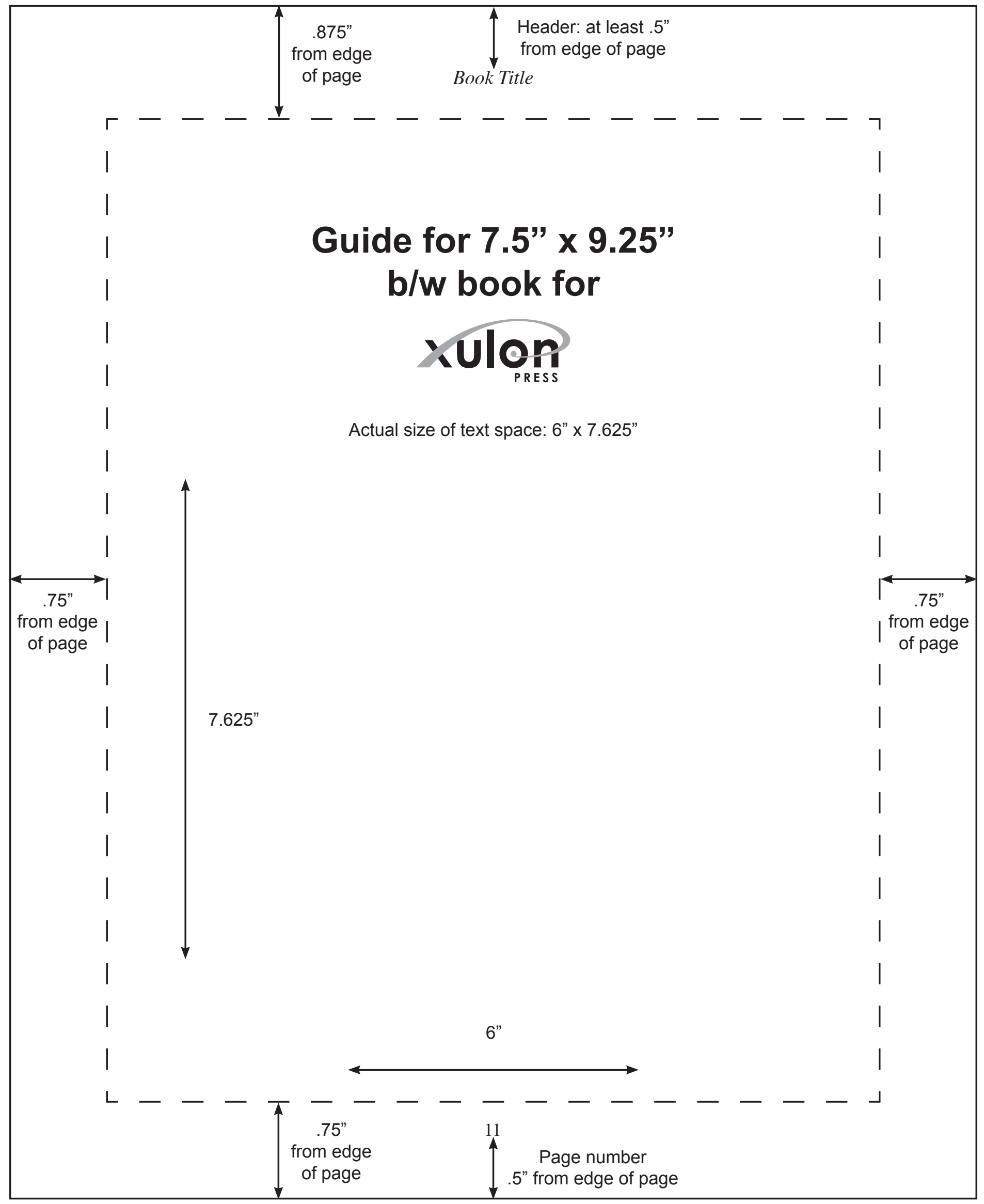




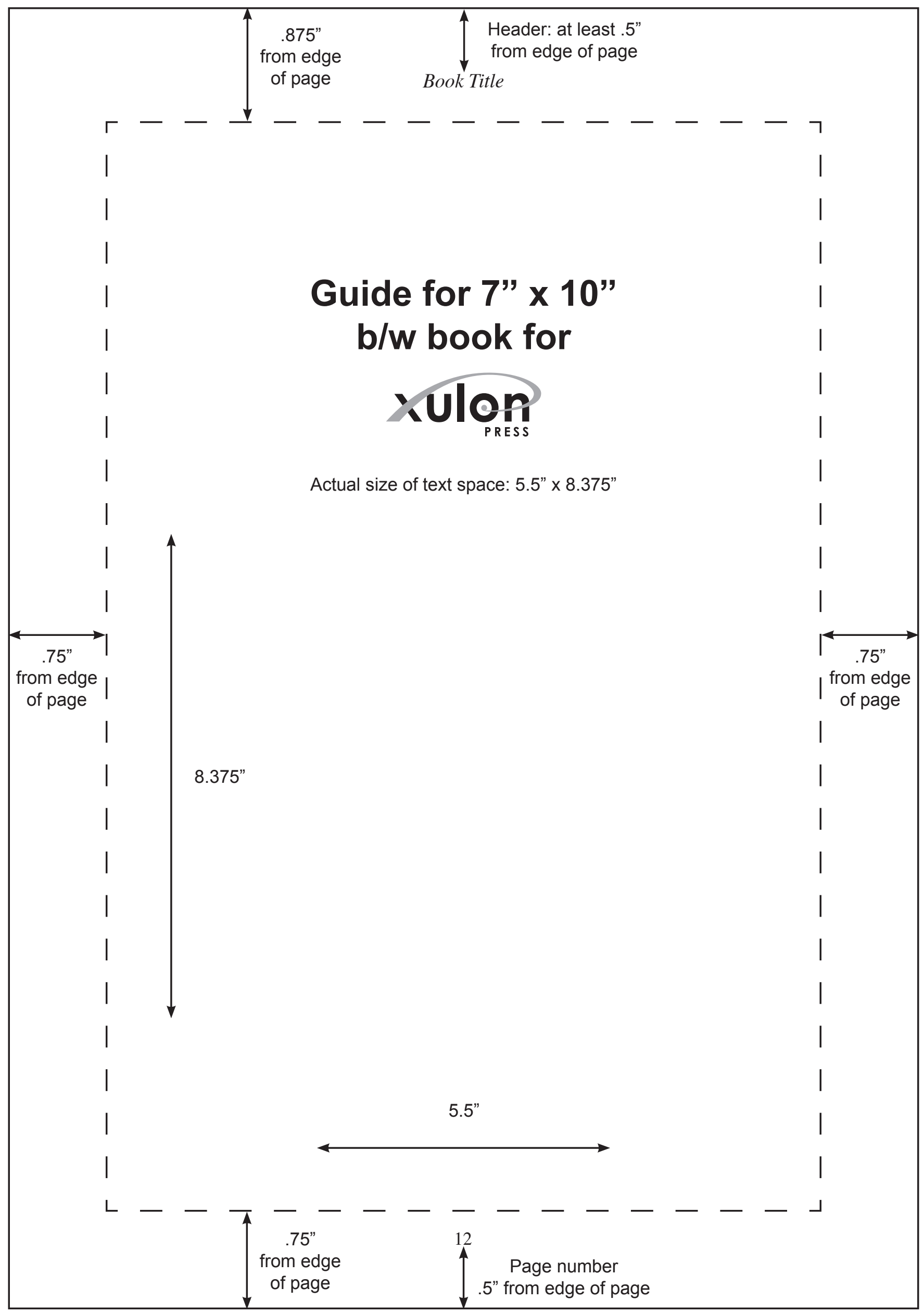




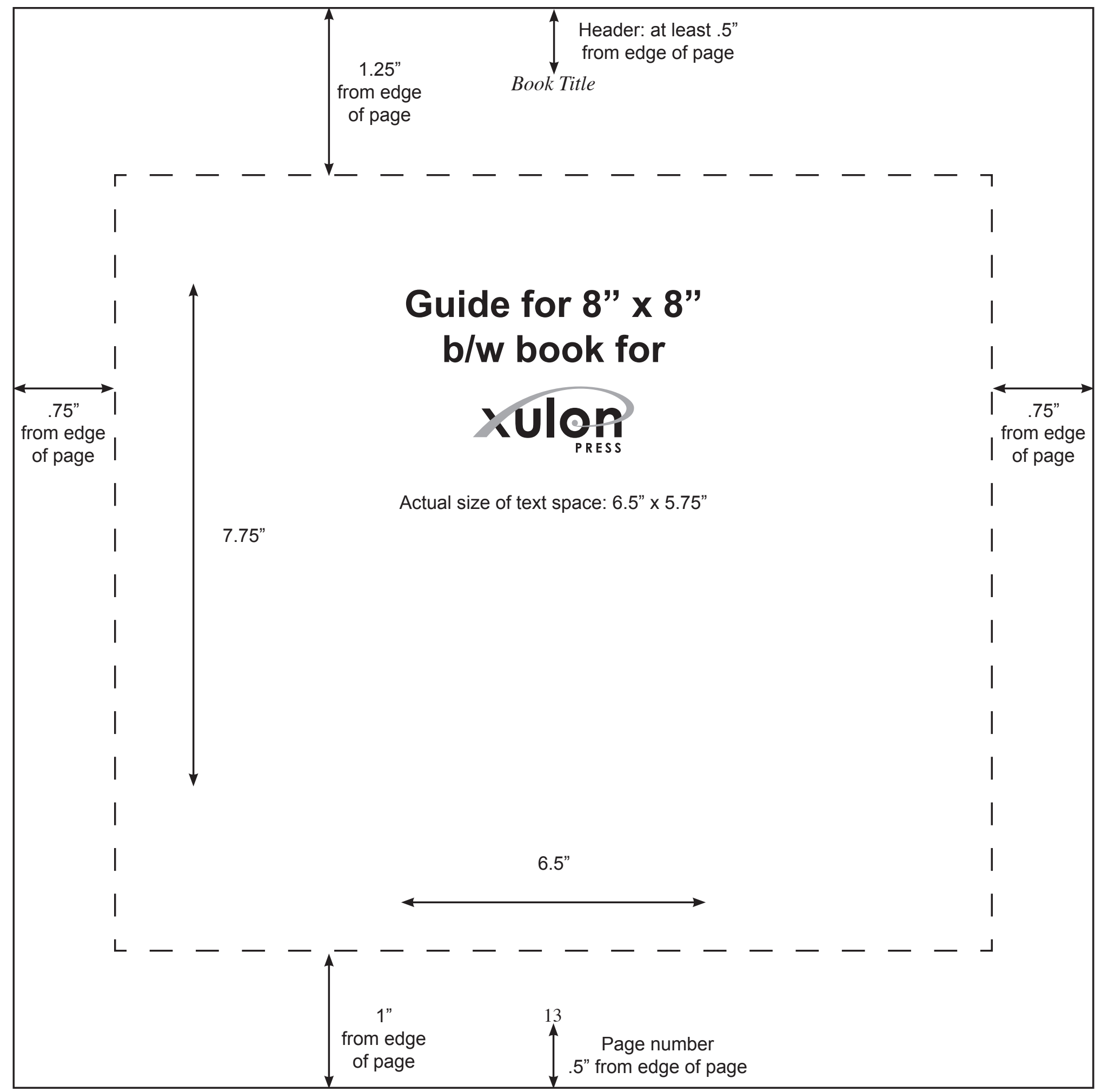




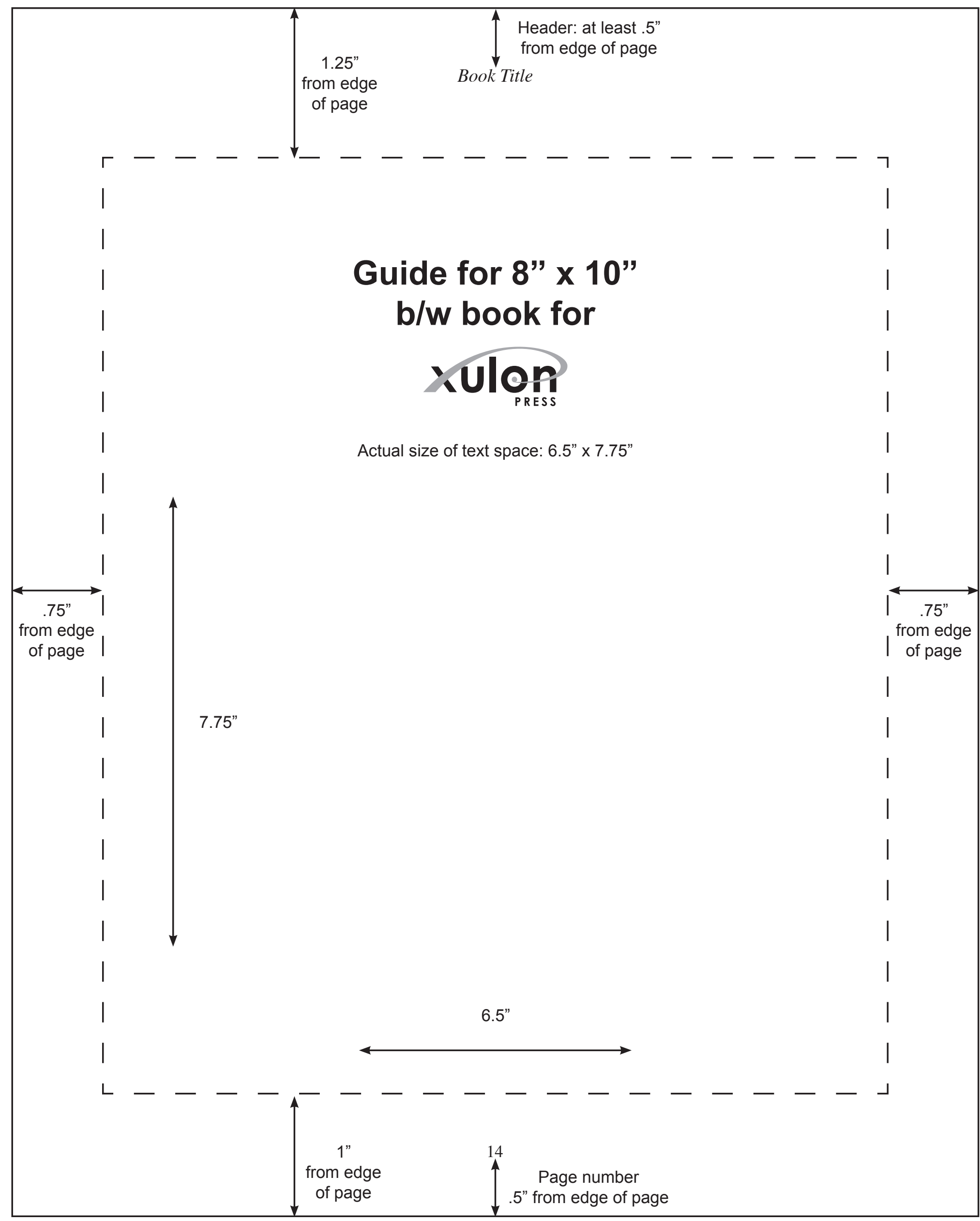




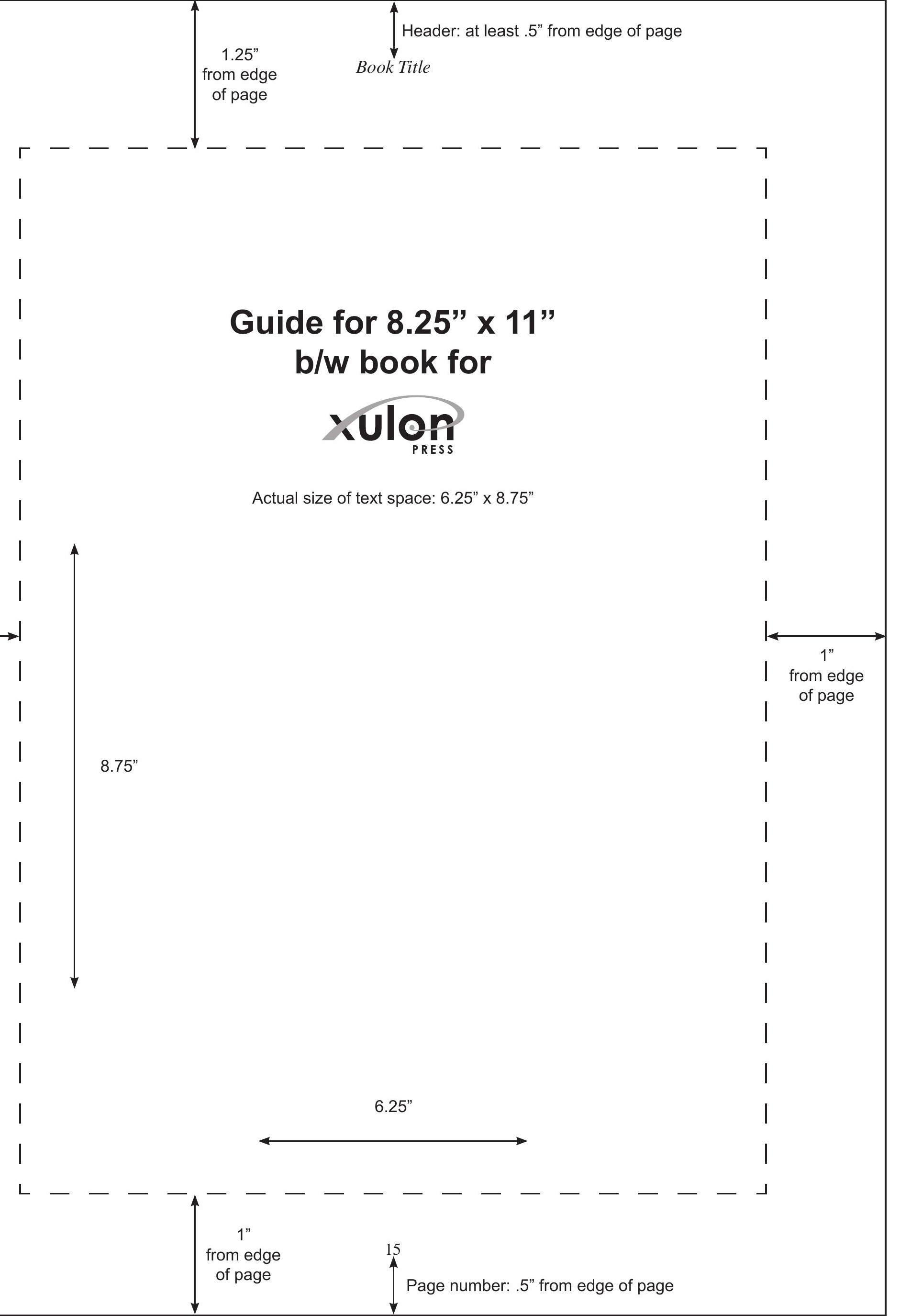


of page

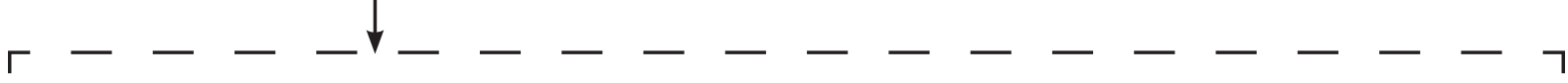

\section{Guide for 8.268" x 11.693" b/w book for xulon}

Actual size of text space: $6.268 "$ x 9.443"

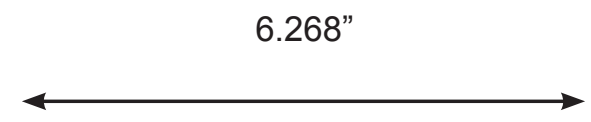


$1.25 "$

from edge

of page

Guide for 8.5" x 11" b/w book for

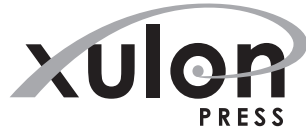

Actual size of text space: $6.5 " \times 8.75 "$

8.75"

$6.5 "$

$1 "$

from edge of page of page

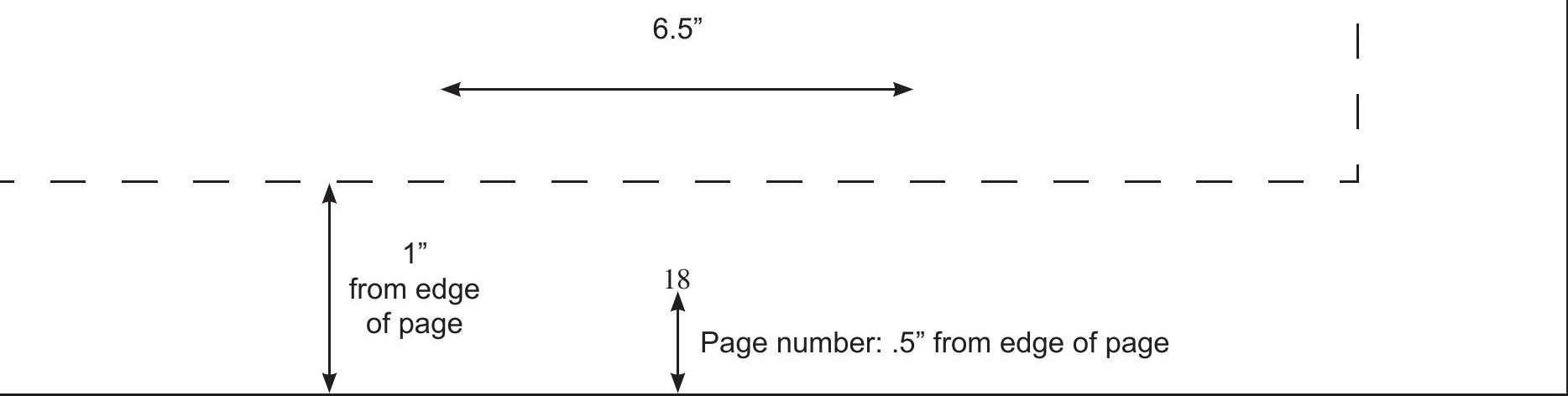

